\section{Primary malignant melanoma of the oesophagus with a left atrial metastasis}

\author{
Massimo Chello, Antonietta R Marchese, \\ Antonio Panza, Pasquale Mastroroberto, \\ Francesco Di Lello
}

\begin{abstract}
A 75 year old man with an eight month history of dysphagia and weight loss underwent pericardiocentesis for a massive pericardial effusion. The echocardiogram showed a mass in the left atrium, and computed tomography also showed distal oesophageal narrowing, which was found to be due to a malignant melanoma. The left atrial mass, investigated by immunoscintigraphy with technetium-99m labelled monoclonal antibody, was diagnosed as metastatic melanoma. This represents a rare case of primary oesophageal melanoma with left atrial metastasis.
\end{abstract}

(Thorax 1993;48:185-186)

Primary oesophageal melanoma is a rare form of neoplasm that accounts for $0.1 \%$ of all oesophageal tumours. ${ }^{12}$ Since its first description by Baur in 1906 almost 100 cases have been reported, in only $10 \%$ of which was the melanoma primary. ${ }^{3}$

Malignant melanoma has a high incidence of myocardial and combined pericardial and myocardial metastases, ${ }^{4}$ the frequency of cardiac metastasis increasing with the number of distant metastases.

In this report we present the case of a man with a metastatic malignant melanoma in the left atrium whose primary tumour was in the oesophagus.

\section{Case report}

A 74 year old man was admitted to hospital with clinical signs of cardiac tamponade. Physical examination showed orthopnoea and enlarged, pulsatile neck veins; the liver was palpable $4 \mathrm{~cm}$ below the right costal margin and was also pulsatile. The heart rate was 150 beats/min and the blood pressure $80 / 50 \mathrm{~mm}$ $\mathrm{Hg}$. An electrocardiogram showed atrial fibrillation and small voltage complexes. A chest radiograph showed a considerably enlarged cardiac silhouette and small opacities were noted in the right lower lobe. A two dimensional echocardiogram showed a large pericardial effusion and a large echogenic mass in the left atrium. The results of liver function tests were severely abnormal.
The patient gave an eight month history of mild dysphagia with solid foods, with weight loss of almost $10 \mathrm{~kg}$.

Under echographic guidance an $8 \mathrm{~F}$ pigtail catheter was introduced in the pericardial space through the left costoxiphoid angle, and $2650 \mathrm{ml}$ of bloody fluid was drained in one hour. A sample was sent for chemical and cytological analysis.

Afterwards an echocardiogram showed a residual pericardial effusion of about $400 \mathrm{ml}$, lying mainly in the posterior pericardial space, and improved myocardial contractility. The pigtail catheter was left in place and a further $450 \mathrm{ml}$ of fluid was drained over the next 48 hours. The pericardial fluid did not collect again and the catheter was removed 72 hours later.

Computed tomography identified a $3-4 \mathrm{~cm}$ narrowing of the distal oesophagus, two masses in the lower lobe of the right lung, and a large, non-thrombotic mass in the left atrium.

Endoscopy showed a $4 \mathrm{~cm}$ irregular mass in the distal oesophagus, and a biopsy specimen of this showed malignant melanoma cells. No skin lesions were found at physical examination but atypical epithelial cells were discovered in the pericardial fluid.

The patient was then referred for more detailed assessment and entered an experimental protocol that used ${ }^{99 \mathrm{~m} T c}$ labelled antimelanoma monoclonal antibody (a fab fragment). Whole body and selected regional images were obtained with a gamma camera seven to eight hours after administration of the labelled antibody. There were two areas of increased uptake in the right lung corresponding to the abnormalities on the chest radiograph and the computed tomogram, and a focus of increased radioactivity in the heart as well as in the distal oesophagus (figure).

No surgical treatment was contemplated owing to the advanced stage of the disease, and the patient was referred for medical treatment.

\section{Discussion}

Primary melanoma of the oesophagus accounts for $0 \cdot 1 \%$ of all primary oesophageal malignancies. There are no clinical differences from other forms of oesophageal cancer, and the histological diagnosis is often difficult, the submucosal site of the tumour being responsible for false negative histological and cytological appearances. ${ }^{5}$

The diagnostic criteria of a primary form of melanoma were clearly defined by Allen and Spitz ${ }^{6}$ as: (1) typical melanoma structure and presence of melanin; (2) origin from squamous epithelium with junctional activity; and (3) junctional activity with melanotic cells in the adjacent epithelium.

The prognosis is extremely poor with a four year survival of $4 \%$. Malignant melanoma and oesophageal and breast cancer have the highest rate of metastasis affecting the heart, ${ }^{78}$ cardiac metastases occurring in up to $60 \%$ of patients with known melanoma. ${ }^{9}$ The

\footnotetext{
Address for reprint requests: Dr M Chello, Via S 80128 Napoli, Italy

Accepted 15 April 1992

Department of Surgery, Medical M Chello

A R Marchese

A Panza
} 


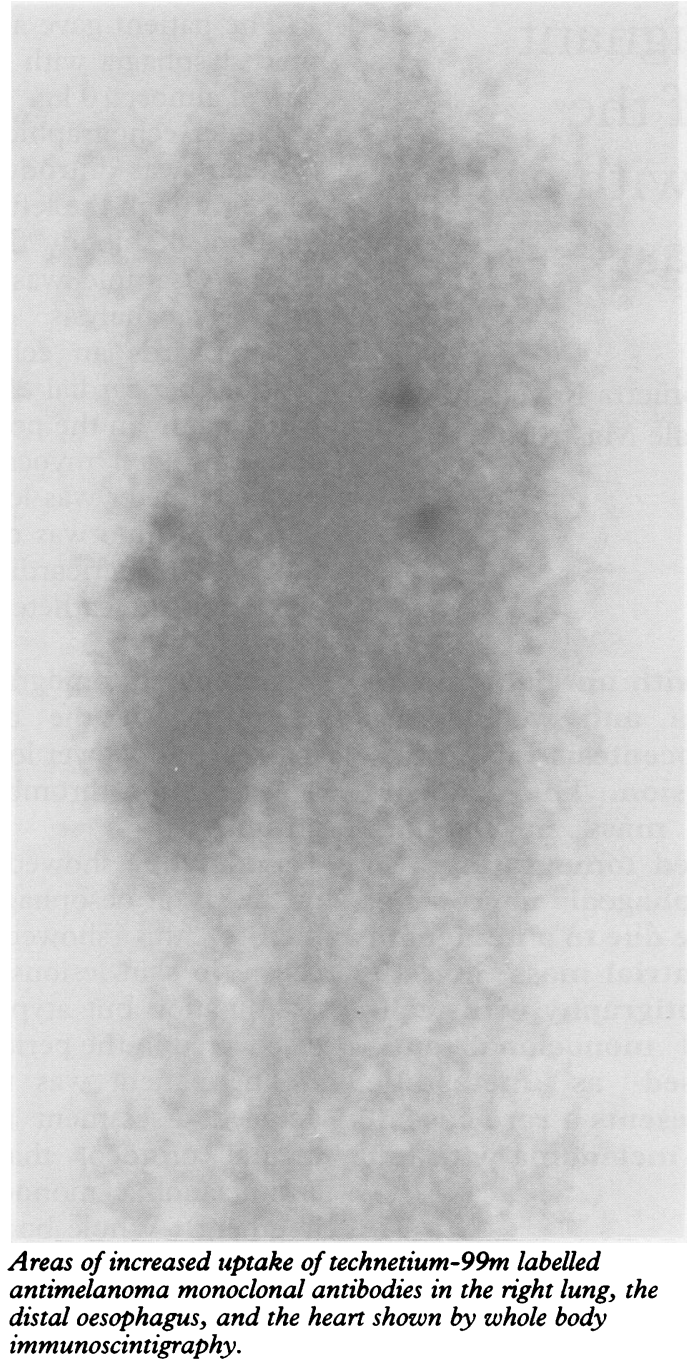

diagnosis of cardiac metastasis is often difficult during life ${ }^{8}$ and in most cases it is discovered at necropsy.

As suggested by Waller, ${ }^{10}$ in patients with melanoma and without previously known heart disease one or more of the following signs suggest cardiac metastasis: (1) acute pericarditis; (2) pericardial effusion; (3) cardiac tamponade or constriction; (4) second or third degree atrioventricular block; (5) congestive heart failure.
The antibody imaging procedure represents a sensitive and relatively simple method for detecting the presence and extent of spread of malignant disease. ${ }^{112}$ The basis of this technique is the presence of a highly specific tumour associated antigen, the oncofetal antigen P97, which is strongly expressed on most melanoma cells, but only weakly on normal cells of human adult tissue. Consequently, by using a specific monoclonal antibody labelled with technetium- $99 \mathrm{~m}$, metastatic foci can be detected by simple immunoscintigraphy. As with most diagnostic imaging procedures, the size of metastatic lesions is an important variable that influences imaging efficiency. ${ }^{13}$ When analysed by size, lesions of over $2 \mathrm{~cm}$ are seen more frequently than lesions of $2 \mathrm{~cm}$ or less. Nevertheless, this antibody imaging procedure represents a useful addition to routine chest radiographs and computed tomograms.

1 Chalkiadakis G, Wilhm JM, Morand G, Weill-Bousson M, Witz JP. Primary malignant melanoma of the esophagus. Ann Thorac Surg 1985;39:472-5.

2 Turnbull AD, Rosen P, Goodner JT, Beattle ES. Primary malignant melanoma of the esophagus other than typical epidermoid carcinoma Ann Thorac Surg cal epidermoid

3 Go ATS, Zirkin R. Primary malignant melanoma of the esophagus: a case report with endoscopic and electron esophagus: a case report with endoscopic and electron

4 Karwinski B, Swendsen E Trends in cardiac metastasis. APMIS 1989;97:1018-24.

5 Lucev M, Turi V, D'Abrosca F, Gerosa E, Villani L. Primary melanoma of the esophagus. Minerva Chirurgica 1990;45:195-7.

6 Allen AC, Spitz S. Malignant melanoma: clinico-pathological analysis of criteria for diagnosis and prognosis. Cancer 1953;6:1-45.

7 Gassman HS, Meadows R, Baker LA. Metastatic tumors of the heart. Am $\mathcal{F}$ Med 1955;19:357-65.

8 Skhatsabaja LV. Secondary malignant lesion of the heart and pericardium in neoplastic disease. Oncology 1986;43:103-6.

9 Glancy DL, Roberts WC. The heart in malignant melanoma, a study of 70 autopsy cases. Am 7 Cardiol melanoma, a study

10 Waller BF, Gottdiener JS, Virmani R. The "charcoal heart": melanoma of the cor. Chest 1980;77:671-6.

11 Lamki LM, Zukiwsi L, Shanken LJ, Legha SS, Bejamin RS, Plager CE, et al. Radioimaging of melanoma using ${ }_{99} \mathrm{~T}$ c labeled $\mathrm{Fab}$ fragment reactive with high molecular weight melanoma antigen. Cancer Res 1990;50 (suppl 3):904-8.

12 Cerny T, Owens SE, Mackenzie SA. Immunoscintigraphy with ${ }^{99 m} \mathrm{Tc}$ labeled fragment of an antimelanoma monoclonal antibody $(225 \cdot 28 S)$ in patients with metastatic malignant melanoma. Eur f Nuc Med 1987;13:130-3.

13 Salk $D$ and the Multicenter Study Group. Technetium labeled monoclonal antibodies for imaging metastatic melanoma. Results of a multicenter clinical study. Semin melanoma. Results of 